\title{
Giant Pulses from Two Pulsars
}

\author{
Ashok K. Singal \\ Astronomy and Astrophysics Division, Physical Research Laboratory, \\ Navrangpura, Ahmedabad 380 009, India \\ P. K. Manoharan \\ Radio Astronomy Centre, Post Box 8, Udhagamandlam - 643001, India
}

Richard G. Strom

NFRA, Radio Observatory, PO Box 2, 7990 AA Dwingeloo, Netherlands

\begin{abstract}
We present observational results from long term monitoring of giant pulses from two pulsars. One of the pulsars (PSR 0950+08) has been observed simultaneously at three different observatories, at different radio frequencies. There is evidence to suggest that the giant pulse phenomenon is intrinsic to the pulsar.
\end{abstract}

Until now giant pulses have been observed from only a couple of pulsars, namely the Crab pulsar (PSR 0531+21, see Lundgren et al. 1995) and one or two millisecond pulsars (in particular, PSR 1937+21, Cognard et al. 1996). Cognard et al. (1996) discussed about the earlier observations of a few dozen strongest pulsars and arrived at the conclusion that with the exception of Crab pulsar and PSR 1937+21, pulses much stronger than 10 times the mean have not been observed before, even rarely, and that pulsars with giant pulses are quite uncommon. Here we report the observations of a large number of giant pulses from two longer period pulsars, PSR 0950+08 $(P=253 \mathrm{~ms})$ and PSR $1133+16(P=1.18 \mathrm{~s})$, and now it appears that the phenomenon is not limited to short period pulsars alone.

Our data for these two pulsars comes primarily from more than a year of daily-half-an-hour monitoring with the Rajkot Radio Telescope at $103 \mathrm{MHz}$. These data show that giant pulse activity fluctuates wildly with changes in both strength and frequency of occurrence. While many observations (daily stretches of half an hour) show strong giant pulses, others over similar stretches of time show practically none. Even on days when there is a strong giant pulse activity, individual giant pulses seem to occur quite randomly. Though sometimes a few giant pulses may follow each other in quick succession, at least in more than two thirds of the cases they appear alone and where immediately next to a giant pulse the neighbouring pulse may be more than an order of magnitude weaker. Often there are gaps lasting for many pulse periods between two successive giant pulses.

We have also observed these two pulsars with the Ooty Radio Telescope at $327 \mathrm{MHz}$ for many sessions lasting over a few hours. Further PSR 0950+08 was 
also observed with the Westerbork Synthesis Radio Telescope (WSRT) using the new Pulsar Machine (PuMa) at $297 \mathrm{MHz}$ for a couple of sessions and some of these observations were made at simultaneous times at the three places. In both WSRT and Ooty observations a trend similar to as seen at Rajkot is noticed. It seems that both the activity and strength of giant pulses may vary on 1-2 hour time scales at these frequencies. There are stretches where the pulses almost disappear, to be followed within several hours by pulses as much as two orders of magnitude above the average pulse strength.

These time scales appear to be too short for either the refractive or diffractive interstellar scintillation, and we see a similar pattern at frequencies which differ by a factor of three. It is likely that giant pulses comprise one or more micro-pulses which may be intrinsically extremely bright (Hankins 1971). We see that many individual giant pulses observed in 0950+08 with the WSRT remain unresolved at millisecond time scales. This points to an origin of this giant pulse activity in an intrinsic phenomenon within the pulsar.

From the data analysis to date (based upon the simultaneous observations at $103 \mathrm{MHz}$ at Rajkot, at $297 \mathrm{MHz}$ at WSRT and at $327 \mathrm{MHz}$ at Ooty) there does not appear to be an obvious correlation between the occurrence of individual giant pulses, or even of pulse strength when averaged over minute time scales. It may well be that giant pulse activity is not very broadband, and indeed the WSRT PuMa data shows decorrelation of individual pulses within the $10 \mathrm{MHz}$ band. Further analysis of these joint data sets is still in progress which may reveal additional information.

GMRT could be an ideal instrument for a detailed study of giant pulses from these pulsars. Since these are relatively strong pulsars, one could even select different sub-sets of GMRT antennas at different frequency bands for a simultaneous study of giant pulses at two or more radio frequencies, which could help us in ascertaining their origin, i.e. an intrinsic phenomenon vis-a-vis an interstellar refraction scintillation effect.

\section{References}

Lundgren, S. C., Cordes, J. M., Ulmer, M., Matz, S. M., Lomatch, S., Foster, R. S., \& Hankins, T. 1995, ApJ, 453, 433

Cognard, I., Shrauner, J. A., Taylor, J. H., \& Thorsett, S. E. 1996, ApJ, 457, L81

Hankins, T. H. 1971, ApJ, 169, 487 